\title{
Soil pH mediates the balance between stochastic and deterministic assembly of bacteria
}

\author{
Binu M. Tripathi ${ }^{1} \cdot$ James C. Stegen $\mathbb{D}^{2} \cdot$ Mincheol Kim ${ }^{1} \cdot$ Ke Dong ${ }^{3} \cdot$ Jonathan M. Adams ${ }^{4} \cdot$ Yoo Kyung Lee ${ }^{1}$
}

Received: 30 March 2017 / Revised: 1 February 2018 / Accepted: 5 February 2018 / Published online: 7 March 2018

(c) The Author(s) 2018. This article is published with open access

\begin{abstract}
Little is known about the factors affecting the relative influences of stochastic and deterministic processes that govern the assembly of microbial communities in successional soils. Here, we conducted a meta-analysis of bacterial communities using six different successional soil datasets distributed across different regions. Different relationships between $\mathrm{pH}$ and successional age across these datasets allowed us to separate the influences of successional age (i.e., time) from soil $\mathrm{pH}$. We found that extreme acidic or alkaline $\mathrm{pH}$ conditions lead to assembly of phylogenetically more clustered bacterial communities through deterministic processes, whereas $\mathrm{pH}$ conditions close to neutral lead to phylogenetically less clustered bacterial communities with more stochasticity. We suggest that the influence of $\mathrm{pH}$, rather than successional age, is the main driving force in producing trends in phylogenetic assembly of bacteria, and that $\mathrm{pH}$ also influences the relative balance of stochastic and deterministic processes along successional soils. Given that $\mathrm{pH}$ had a much stronger association with community assembly than did successional age, we evaluated whether the inferred influence of $\mathrm{pH}$ was maintained when studying globally distributed samples collected without regard for successional age. This dataset confirmed the strong influence of $\mathrm{pH}$, suggesting that the influence of soil $\mathrm{pH}$ on community assembly processes occurs globally. Extreme $\mathrm{pH}$ conditions likely exert more stringent limits on survival and fitness, imposing strong selective pressures through ecological and evolutionary time. Taken together, these findings suggest that the degree to which stochastic vs. deterministic processes shape soil bacterial community assembly is a consequence of soil $\mathrm{pH}$ rather than successional age.
\end{abstract}

These authors contributed equally: Binu M. Tripathi and James C. Stegen.

Electronic supplementary material The online version of this article (https://doi.org/10.1038/s41396-018-0082-4) contains supplementary material, which is available to authorized users.

$\triangle$ Jonathan M. Adams

j.m.adams@cranfield.ac.uk

$\triangle$ Yoo Kyung Lee

yklee@kopri.re.kr

1 Korea Polar Research Institute, Incheon 21990, Republic of Korea

2 Pacific Northwest National Laboratory, 902 Battelle Boulevard, P. O. Box 999, Richland, WA 99352, USA

3 Department of Biological Sciences, College of Natural Sciences, Seoul National University, 1 Gwanak-ro, Gwanak-gu, Seoul 08826, Republic of Korea

4 School of Water, Energy and Environment, Cranfield University, Cranfield MK43 OAL, UK

\section{Introduction}

Understanding the fundamental ecological processes that shape the assembly of microbial communities is a major challenge in microbial ecology [1]. The assembly of microbial species in a local community is influenced by two types of ecological processes, namely deterministic and stochastic [2, 3]. Deterministic processes include ecological selection imposed by abiotic and biotic factors, which influence organismal fitness and thereby determine the composition and relative abundance of species $[4,5]$. Stochastic processes, in contrast, involve random birth, death, and dispersal events that result in patterns of species composition indistinguishable from those produced by random chance alone [6, 7]. Both deterministic and stochastic processes act concurrently to regulate the assembly of ecological communities [8-11]. However, variation in strength of ecological selection and rates of dispersal influence the relative importance of deterministic and stochastic processes across time, space, and from one system to another [12-14]. 
An open question is the degree to which differences in the balance between stochastic and deterministic processes are driven by differences between environments rather than time itself. Successional soils represent a series of soils of different ages with varying abiotic and biotic characteristics [15]. The pedogenesis (soil development) processes along successional soils results in directional change in nutrient content and often, but not always, lead to declines in $\mathrm{pH}$ [16-19]. Over the past decade, several studies have characterized the shifts in soil microbial communities along successional soils — on both shorter [20-25] and longer [26, 27] time scales. However, relatively little is known about the underlying ecological processes that govern the assembly of microbial communities in successional soils $[13,21,28]$. One limitation is that in any single dataset or system, environmental conditions often vary systematically with time such that these variables are confounded. Bringing together a set of successional systems that differ in the relationship between soil age and environmental properties is ideal for disentangling the relative contributions of the environment vs. time.

Recent studies have indicated that in early successional soils, bacterial community assembly is largely governed by stochastic processes, with the relative importance of deterministic processes increasing progressively in later successional soils $[13,28]$. However, it is still not clear what underlying environmental factors or community processes may be changing between early and late successional soils to produce this increased importance of determinism. For instance, it is well known that soil $\mathrm{pH}$ is very important in determining variation in bacterial community structure and diversity [29-33]. As pH often changes along successional soils [17], this could be the main cause of the observed trends in bacterial community assembly and also influence the relative importance of stochastic and deterministic processes across successional soils. Alternatively, time itself could be the driver wherein biological interactions change through time to alter the balance between stochastic and deterministic processes. To examine whether differences in soil $\mathrm{pH}$ (and other environmental variables) or time were the primary drivers of shifts in the stochastic-deterministic balance, we carried out a meta-analysis across a range of different successional soils, not all of which show the same trend in soil $\mathrm{pH}$ over time. This is a powerful design whereby successional age does not consistently co-vary with $\mathrm{pH}$. Because successional age and $\mathrm{pH}$ do not confound each other, their relative influences can be evaluated. We applied an ecological null modeling approach to analyze the community assembly processes with the following questions:

1. Are there a predictable differences in bacterial community assembly across successional soils, regardless of differences in local environmental constraints? If community assembly processes are driven primarily by the amount of time a local community has had to develop, we expect ecological selection to be relatively weak during early succession and to increase in strength with time. In this case, the influence of stochastic processes relative to deterministic processes should decrease with successional age. On the other hand, if niches become more available as nutrients accumulate in later successional soils [17], a broader range of species may be able to establish. This scenario would lead to an increase in the relative influence of stochastic processes. In either case, there should be a directional shift in the balance between stochastic and deterministic processes regardless of differences in the abiotic environment.

2. Does soil $\mathrm{pH}$ explain differences in bacterial community assembly processes along successional soils, irrespective of successional age? An earlier conceptual model suggested that lack of strong environmental filtering in early successional soils leads to dominance of stochastic processes [13]. This suggests that time itself is not a driver of community assembly processes, and that assembly processes change through time because of changes to the abiotic environment. In this case, we expect that the balance between stochastic and deterministic processes will vary with $\mathrm{pH}$ (or other environmental variables) regardless of successional age.

\section{Materials and methods}

\section{Datasets}

For studying the bacterial community assembly and relative influence of ecological processes along successional soils, we compiled six datasets of varying soil $\mathrm{pH}$ gradients between early and late successional soils (Table 1 ). The four shorter-term ( 150 years) datasets of successional soils were: Austre Lovénbreen Glacier (AL) [22], Midtre Lovénbreen Glacier (ML) (newly presented in this study), Damma Glacier (DM) [24], and Easton Glacier (ES) [23]. The two longer-term datasets of successional soils were 120,000-year-old Franz Josef Glacier (FJ) [26], and 4000year-old Wilderness Park sand-dune soil succession (SD) [27]. Except for the ML site, the sequence and metadata were obtained directly from authors or downloaded from the public repository. In the case of ML, soil samples were collected along the glacier chronosequence in summer 2014. A total of 39 samples were collected from three transects from the glacier terminus to the edge of the foreland moraine (for detailed information on sampling, see ref. [34]). The 


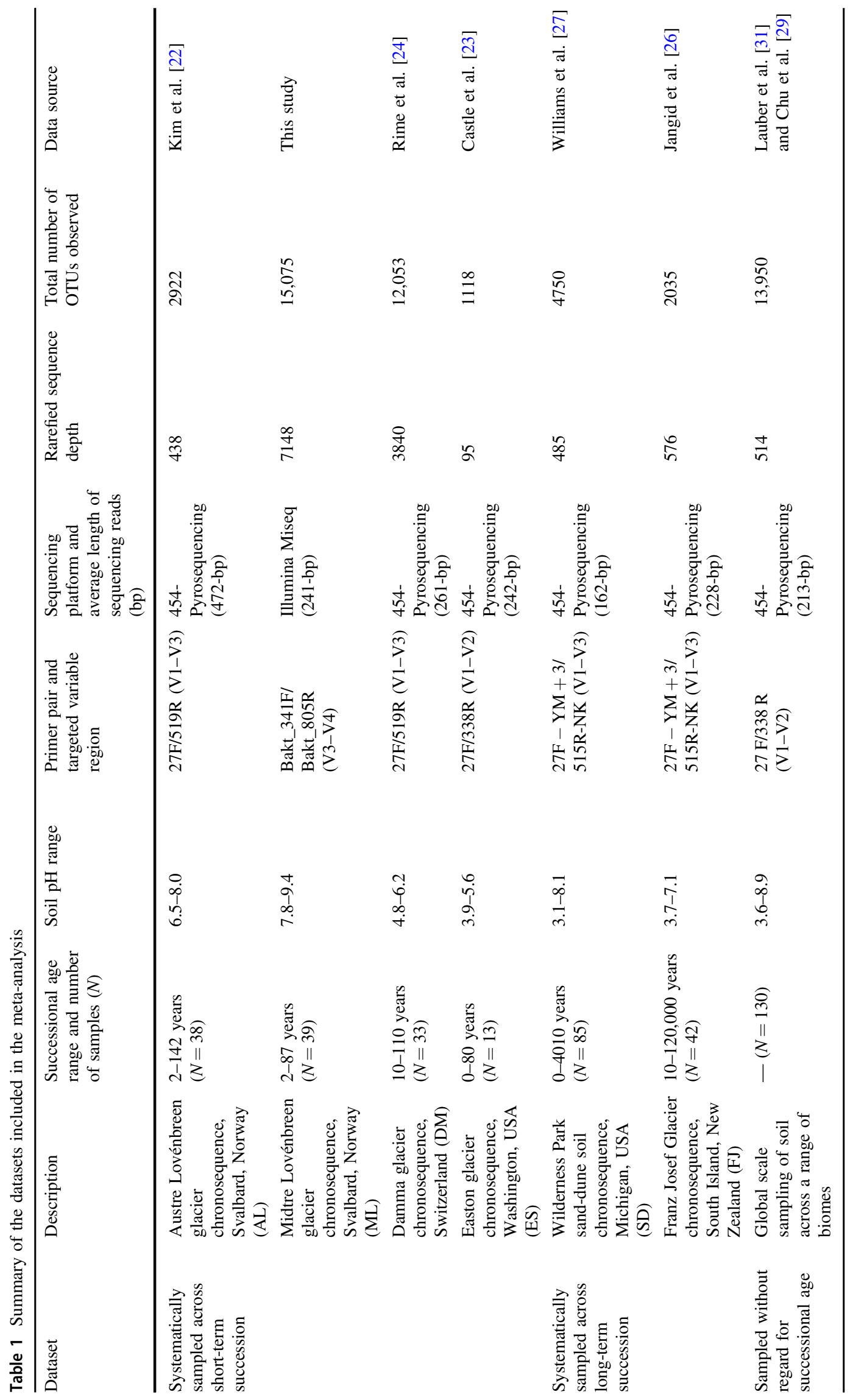


successional datasets showed a strong influence of $\mathrm{pH}$ and little influence of successional age. Given that $\mathrm{pH}$ had a much stronger association with community assembly processes than did successional age, we evaluated whether the inferred influence of $\mathrm{pH}$ was maintained in a broader range of soil ecosystems. To do so, we studied 130 globally distributed soil samples that were collected without regard for successional age [29, 31]. Like all soils, these have a successional history, but this information is not available in the global metadata, and they were not systematically collected with respect to successional stage. Hence, these samples represent a heterogeneous set of soils that differ in climate, vegetation, etc., and we assume they also differ in successional history. Despite this heterogeneity-and despite not being able to control for successional stage- the effect of $\mathrm{pH}$ was clearly observed. We suggest that better controlling for potentially confounding factors (e.g., successional stage) may reveal an even stronger influence of $\mathrm{pH}$.

\section{DNA extraction and sequencing}

The MoBio Power Soil DNA extraction kit (MoBio Laboratories, Carlsbad, CA, USA) was used to extract the DNA from samples collected along the ML chronosequence. The extracted DNA samples were sent to Macrogen Incorporated (Seoul, Korea) for sequencing. The V3 and V4 region of the 16S rRNA gene was amplified using bacterial primers Bakt_341F and Bakt_805R [35]. The resulting amplicons were sequenced using 300-bp pair-end Illumina MiSeq system (Illumina, San Diego, CA, USA). The 16S rRNA gene sequence data from the ML chronosequence was deposited in the MG-RAST server under project ID mgp21131 (http:// metagenomics.anl.gov/linkin.cgi?project=mgp21131).

\section{Sequence processing}

The sequence datasets were analyzed separately as these datasets contained sequences from different variable $16 \mathrm{~S}$ rRNA gene regions (Table 1). The initial quality filtering steps were different for pyrosequencing and Illumina datasets. All the pyrosequenced datasets, except ES and SD, were quality filtered in Mothur [36]. Briefly, sequences with barcode ambiguities, with read length $<150 \mathrm{bp}$, and with average quality score $<25$ were removed. The obtained sequences of ES and SD sites were already quality filtered by authors (for detailed information on quality filtering, see refs. [23, 27]). The Illumina sequences from the ML site were quality filtered by following the error correction strategy of Schirmer et al. [37]. Briefly, the paired-end sequences were quality trimmed (Sickle) and error corrected (BayesHammer) before being assembled using PANDAseq [38].

The quality filtered sequences were aligned against SILVA alignment version 123 (http://www.arb-silva.de/).
Chimeric sequences were detected and removed via the Chimera UCHIME algorithm in de novo mode contained within mothur [39]. The operational taxonomic units (OTUs) were clustered using the average neighbor clustering algorithm with a threshold of $97 \%$ sequence similarity. Phylogenetic null models can be sensitive to sequencing errors, so to avoid spurious results due to sequencing errors, singleton OTUs were not used in subsequent analyses. Finally, each OTU table was rarefied to equal sequence depth (Table 1) by random subsampling to minimize the effect of sequence depth variations among samples.

\section{Phylogenetic analysis}

Aligned sequences of representative OTUs were used to construct a maximum-likelihood tree in FastTree [40]. To test for phylogenetic signal, we first calculated environmental optima for all OTUs with respect to soil $\mathrm{pH}$ by following the procedure described by Stegen et al. [2]. Briefly, for each OTU its relative abundance-weighted mean value was calculated for soil $\mathrm{pH}$. To calculate the abundance-weighted mean for a given OTU, we first found all samples in which that OTU was present. We then found the abundance-weighted mean $\mathrm{pH}$ of all those samples. To do so, in the calculation of mean $\mathrm{pH}$ we weighted each $\mathrm{pH}$ value by the abundance of the OTU in the associated sample. This procedure was repeated for each OTU, and the resulting value was used as a rough estimate of that OTU's $\mathrm{pH}$ optimum. Then, between-OTU differences in $\mathrm{pH}$ optima were calculated as Euclidean distances. Finally, we used Mantel correlograms to measure the correlation coefficients between differences in $\mathrm{pH}$ optima and phylogenetic distances [3, 9], and significance of these correlations was assessed using 999 permutations with Bonferroni correction. We also used Huisman-Olff-Fresco (HOF) hierarchic regression models [41] implemented in the $R$ package "eHOF" [42] as an alternative method to calculate niche optima. The eHOF approach selects the best-fit out of the pre-determined model types (seven types of hierarchical models) for each OTU, using Akaike information criterion and bootstrapping to stabilize the model choice. We excluded OTUs with type I model fit, because type I model is flat and has no niche optimum.

To evaluate the phylogenetic community assembly, we calculated the standardized effect size measure of the mean nearest taxon distance (SES.MNTD) using the null model "taxa.labels" (999 randomization) in "picante" R package [43]. Lower values of SES.MNTD $(<0)$ indicate phylogenetic clustering (i.e., co-occurring OTUs are more closely related than expected by chance), whereas higher values $(>0)$ indicate phylogenetic over dispersion (i.e., cooccurring OTUs are less closely related than expected by chance) [44]. 
The pairwise phylogenetic turnover between communities was calculated as the mean nearest taxon distance metric (from here on we refer to this as $\beta$ MNTD [2, 45] using "comdistnt" function (abundance.weighted = TRUE) from the "picante" R package [43]). Furthermore, to infer community assembly processes, we implemented a previously developed null modeling approach [3, 9, 13, 46]. To do so, we first calculated the $\beta$-nearest taxon index $(\beta \mathrm{NTI})$, which is the difference between observed $\beta$ MNTD and mean of the null distribution of $\beta$ MNTD normalized by its standard deviation. $\beta$ NTI values $<-2$ indicate significantly less than expected phylogenetic turnover (homogeneous selection) [13], whereas $\beta$ NTI values $>+2$ indicate significantly more than expected phylogenetic turnover (variable selection) [13].

If the observed $\beta M N T D$ values does not significantly deviate from the null $\beta$ MNTD distribution $(|\beta N T I|<2)$, this indicates that the observed difference in phylogenetic community composition is not the result of deterministic selection [47], and hence it should be due to dispersal limitation (very low rates of dispersal), homogenizing dispersal (very high rates of dispersal), or is not the result of a single dominant process (i.e., it is "undominated"). To differentiate between these scenarios, we further calculated the Bray-Curtis-based Raup-Crick metric $\left(\mathrm{RC}_{\text {bray }}\right)$ as described by Stegen et al. [9] on pairwise comparisons with $|\beta \mathrm{NTI}|<2$.

The relative contributions of different community assembly processes were estimated following the method originally proposed in Stegen et al. [9] and modified in Stegen et al. [46] and Dini-Andreote et al. [13]. More specifically, the relative contributions of variable and homogeneous selection were estimated as the percentage of pairwise $\beta$ NTI values that fell above +2 and below -2 , respectively. The relative contribution of dispersal limitation was estimated as the percentage of pairwise comparisons with $|\beta N T I|<2$ and $R_{\text {bray }}>+0.95$. The relative contribution of homogenizing dispersal was estimated as the percentage of pairwise comparisons with $|\beta N T I|<2$ and $\mathrm{RC}_{\text {bray }}<-0.95$. Pairwise comparisons that did not fall into any of these categories indicate that no single process dominated community assembly. The undominated fraction was therefore estimated as the percentage of pairwise comparisons with $|\beta N T I|<2$ and $\left|\mathrm{RC}_{\text {bray }}\right|<0.95$. The logic behind this approach and simulation models that support these inferences are provided in Stegen et al. [9, 46] and Dini-Andreote et al. [13].

\section{Statistical analyses}

To test the effect of successional age and soil $\mathrm{pH}$ on SES. MNTD across all datasets, we used a generalized additive mixed model (GAMM). GAMM was fitted using the "gamm" function of "mgcv" R package [48]. We used cubic regression spline smoothers for each explanatory variable in the GAMM, with study site as a random factor.

To assess the relative influence of stochastic and deterministic assembly processes across successional soils, we compared all possible pairwise comparisons of $\beta$ NTI values within different successional ages. To further evaluate the variation in community assembly processes along gradients of soil $\mathrm{pH}, \beta \mathrm{NTI}$ values-which are derived from pairwise comparisons-were regressed against Euclidean distance matrices of soil $\mathrm{pH}$. This was done both within and among successional ages. The statistical significance of the resulting comparisons was determined by Mantel tests with 999 permutations. Further, to assess the relationship between phylogenetic turnover and soil $\mathrm{pH}$ or spatial distance after controlling for spatial or soil $\mathrm{pH}$ distance, we performed partial Mantel test with 999 permutations. Similarly, the influence of soil $\mathrm{pH}$ on $\beta \mathrm{NTI}$ was also compared with other environmental variables and spatial distance. These analyses were performed using the "mantel" function of "ecodist" R package [49]. As the library size varied widely between subsampled datasets with lower coverage in some of the datasets (Table 1), we evaluated the effect of library coverage on results by varying the library size and subsampling more and less sequences from datasets with lower and higher library coverage, respectively.

\section{Results}

\section{Phylogenetic signal}

Phylogenetic signal was very similar between the abundance-weighted mean and eHOF approaches. Both methods indicated significant phylogenetic signal across short phylogenetic distances (Figs. S1 and S2). Therefore, we calculated SES.MNTD and $\beta$ NTI because both of these metrics emphasize phylogenetic relationships across short phylogenetic distances. However, there are some caveats related to the use of eHOF. In eHOF approach, model fits can be poor when data are sparse and/or unevenly distributed across the environmental axis, and many OTUs can be removed if the best-fit models are commonly type I, which is flat and has no niche optimum.

\section{Trends in phylogenetic community assembly}

The mean values of SES.MNTD were significantly less than zero in all datasets (Fig. S3; one sample $t$-test, $P<0.05$ ), indicating that in each dataset the bacterial community was more phylogenetically clustered than expected by chance. The GAMM analysis showed that only soil $\mathrm{pH}$ was significantly related to SES.MNTD across all sites, and it 


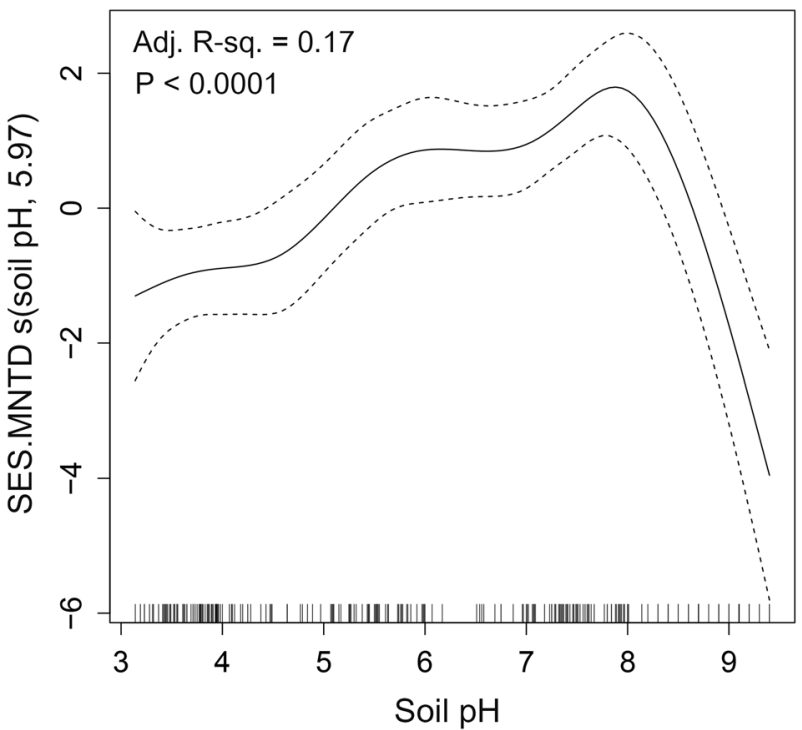

Fig. 1 Effect of soil pH on SES.MNTD of bacterial communities (solid line) across all datasets obtained from generalized additive mixed model (GAMM). The $y$-axis shows the contribution of the fitted centered smooth terms (soil pH, estimated degrees of freedom) to SES. MNTD. Ticks along the $x$-axis indicate the distribution of data for soil $\mathrm{pH}$. The dotted lines represent the upper and lower 95\% confidence intervals

explained $17.2 \%$ of the model deviance with a significant $(P<0.05)$ non-linear fit (Table S1). The effect of successional age on SES.MNTD was non-significant. The SES. MNTD values increased from $\mathrm{pH} \sim 3$ to $\sim 7.8$ and exhibited a sharp decline across $\mathrm{pH}$ values $>7.8$ (Fig. 1). This result indicates that bacterial community assembly was phylogenetically more clustered in more acidic and alkaline soils and phylogenetically less clustered in soils close to neutral $\mathrm{pH}$. As the effect of successional age was not significantly related to SES.MNTD in the datasets that were sampled systematically across successional ages, we further analyzed the SES.MNTD pattern in soils collected globally, and without regard for successional age. These samples spanned a wider range of $\mathrm{pH} 3.6-8.9$ [29, 31]. The SES.MNTD values showed a unimodal pattern along the $\mathrm{pH}$ gradient (Fig. 2; Adj. $R^{2}=0.21, P<0.0001$ ), peaking at close to neutral $\mathrm{pH}$. This result further supports the importance of soil $\mathrm{pH}$ in shaping community assembly processes in soil bacterial communities.

\section{Relative influence of deterministic and stochastic assembly processes}

We examined the relationship between $\beta$ NTI and successional age to infer changes in the relative influences of deterministic and stochastic assembly processes along successional chronosequences. The pairwise comparisons of $\beta N T I$ values within each successional age category indicated various patterns in different datasets (Fig. 3). In AL,

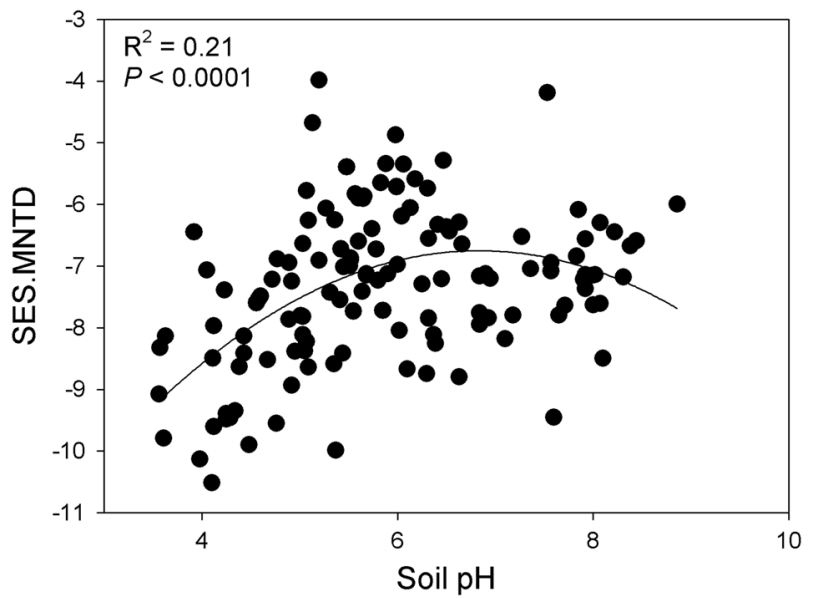

Fig. 2 Relationship between soil $\mathrm{pH}$ and SES.MNTD of bacteria in samples collected across several different biomes [29, 31]

$\mathrm{DM}, \mathrm{FJ}$, and SD chronosequences, the $\beta \mathrm{NTI}$ distributions gradually shifted over the successional ages, from stochastic community assembly $(|\beta \mathrm{NTI}|<2)$ to homogeneous selection $(\beta \mathrm{NTI}<-2)$ (Fig. 3). However, the trend in the $\beta$ NTI distribution over successional ages was opposite in the ML chronosequence, where homogeneous selection was dominant in early successional soils and then shifted towards dominance of stochastic assembly in late successional soils (Fig. 3). In the ES chronosequence, stochastic community assembly remained dominant across all successional ages.

Pairwise comparisons of $\beta$ NTI values both within and among successional ages were significantly correlated to differences in soil $\mathrm{pH}$ (Fig. 4), except in the DM chronosequence. After controlling for spatial distance, soil $\mathrm{pH}$ distance was still significantly correlated with $\beta$ NTI within four sites (Table S2). After controlling for soil $\mathrm{pH}$ differences, spatial distances were significantly correlated with $\beta$ NTI only in AL and DM chronosequences (Table S2). The relationship between soil $\mathrm{pH}$ and $\beta$ NTI remained significant across most of the datasets even when controlled for other measured environmental variables and spatial distance (Table S3). When we combined all possible pairwise comparisons of $\beta$ NTI values from all datasets, the relationship between $\beta$ NTI and difference in soil $\mathrm{pH}$ remained significant (Fig. S4). These results were further supported in a global scale dataset, where we observed a strong correlation between $\beta$ NTI and difference in soil pH (Fig. 5). These results indicate that as the difference in soil $\mathrm{pH}$ increases, there is a transition in bacterial community assembly processes from homogeneous selection, to stochasticity, to variable selection.

We further divided each successional dataset into different soil $\mathrm{pH}$ categories. Within each $\mathrm{pH}$ category pairwise comparisons of $\beta$ NTI showed that the relative influence of homogeneous selection was stronger in highly acidic or alkaline soils (Fig. S5), whereas stochastic community 

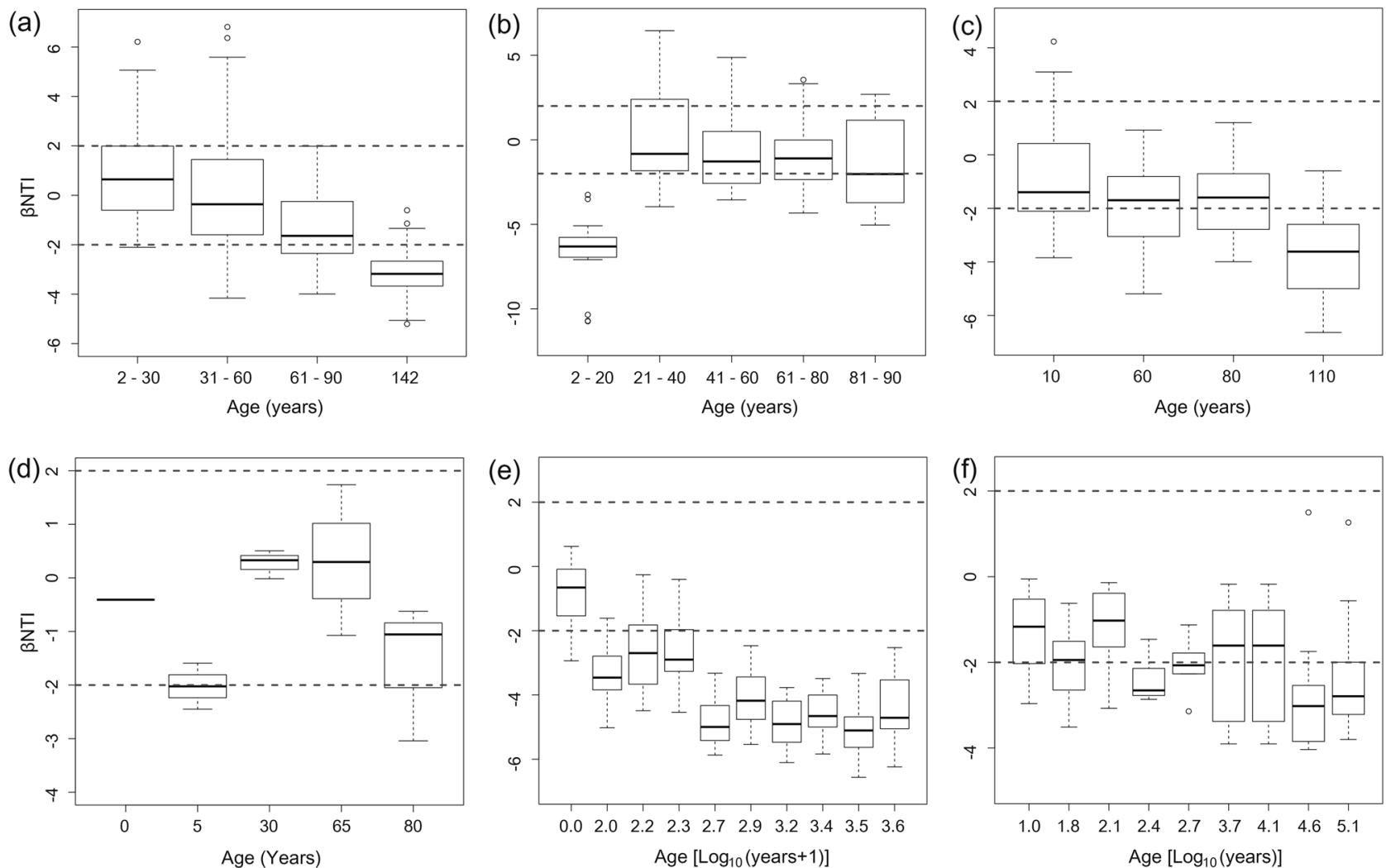

Fig. 3 Patterns of $\beta$ NTI across successional ages in a AL, b ML, $\mathbf{c}$ DM, $\mathbf{d}$ ES, e FJ, and $\mathbf{f}$ SD chronosequences. Horizontal dashed blue lines indicate upper and lower significance thresholds at $\beta \mathrm{NTI}=+2$ and -2 , respectively

assembly was dominant in soils close to neutral $\mathrm{pH}$ (Fig. S5). We found similar results in the global scale dataset (Fig. S6), with dominance of homogeneous selection in more acidic and alkaline soils, and dominance of stochastic assembly in soils close to neutral $\mathrm{pH}$.

\section{Quantitative estimates of assembly processes}

We quantified the relative contributions of each assembly processes over successional ages in all datasets (Fig. 6). The trend in the fraction of homogeneous selection was similar in $\mathrm{AL}, \mathrm{DM}, \mathrm{SD}$, and $\mathrm{FJ}$ chronosequences, which increased with successional age (from $\sim 3$ to $30 \%$ at earliest successional age to $\sim 70-100 \%$ at the latest successional age). Also in these datasets, dispersal limitation (0-50\%), homogenizing dispersal $(0-16 \%)$, and the undominated fraction $(\sim 27-61 \%)$ all showed significant influences in early successional soils; these influences decreased in late successional soils (dispersal limitation: $0-5 \%$, homogenizing dispersal: $0-20 \%$, and undominated: $0-12 \%$ ). In the ML chronosequence, the fraction of homogeneous selection decreased from $100 \%$ in early successional soils to $53.3 \%$ at the late successional soils, whereas the fraction of stochastic assembly increased in late successional soils (dispersal limitation: $21 \%$, homogenizing dispersal: $3 \%$, undominated:
$28 \%$,). In the ES chronosequence, the community was primarily influenced by stochastic assembly, where homogenizing dispersal (100\%) was dominant in early successional soils and assembly was undominated in late successional soils $(66.6 \%)$. The fraction of various assembly processes also varied across $\mathrm{pH}$ categories in all datasets (Fig. S7), with the fraction of deterministic selection processes (homogeneous and variable selection) highest in acidic and alkaline soils, and the fraction of stochastic processes (dispersal limitation, homogenizing dispersal, and undominated) highest in soils close to neutral $\mathrm{pH}$.

\section{Effect of library size on inferred assembly processes}

The effects of library coverage on inferences related to phylogenetic community assembly processes were evaluated by comparing the results at three different randomly subsampled sequence depths (including the original sequence depth) of each dataset. The results showed that except in ES chronosequence, the SES.MNTD and $\beta$ NTI trends were robust (Table S4; Fig. S8). It is therefore likely that observed patterns and the associated inferences were not the result of biases introduced by variation in sequencing depth. Owing to low coverage, increasing the sampled library size in ES chronosequence lead to removal of most 

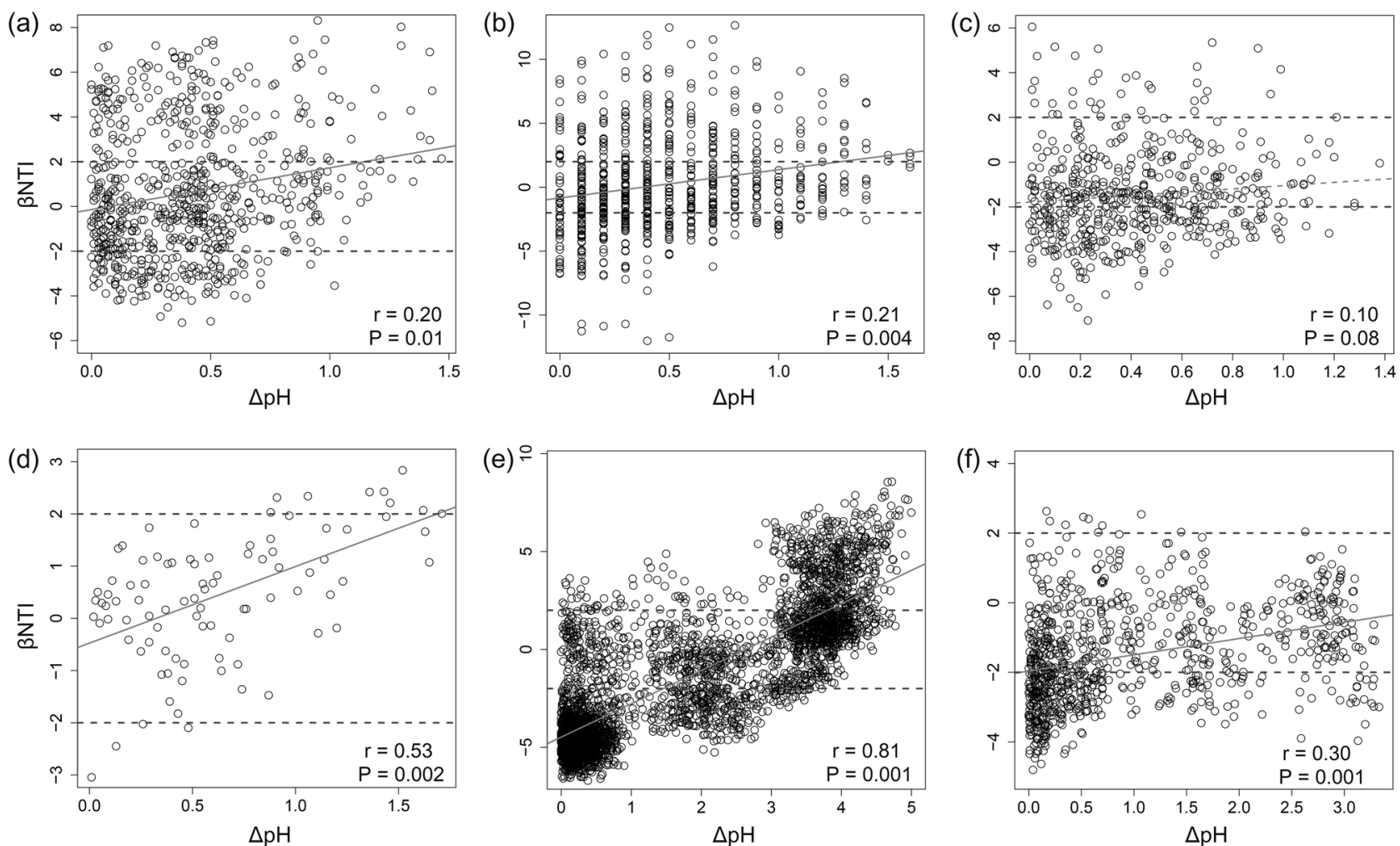

Fig. 4 The relationships between $\beta$ NTI and differences in soil pH for a AL, b ML, $\mathbf{c}$ DM, $\mathbf{d}$ ES, e FJ, and $\mathbf{f}$ SD chronosequences

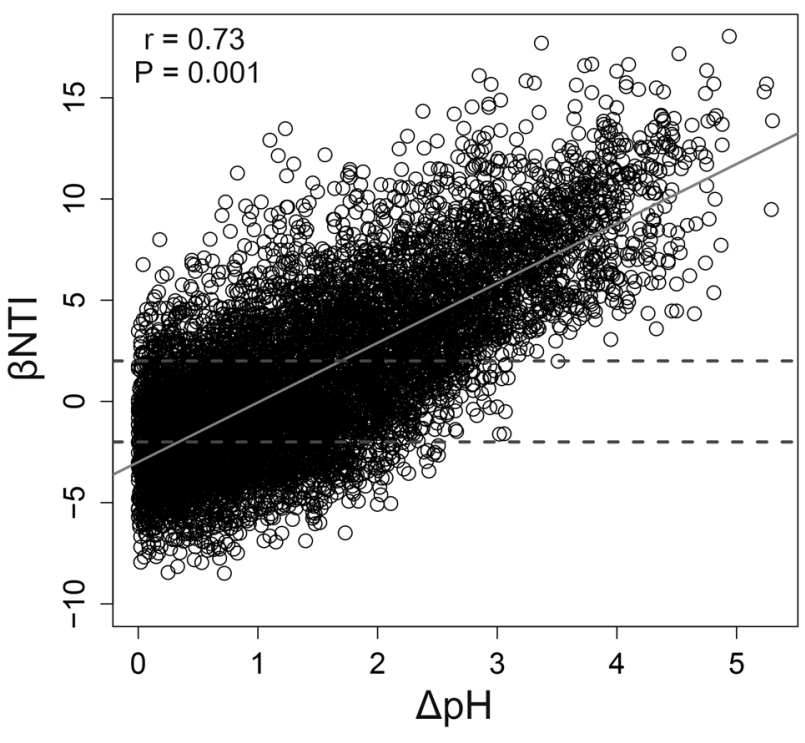

Fig. 5 The relationship between $\beta$ NTI and differences in soil $\mathrm{pH}$ for samples collected at global scale across several different biomes [29, 31]

of the samples from early successional soils ( 0 and 5 years), which also had lower $\mathrm{pH}$ values. The reduced sample size and narrow $\mathrm{pH}$ range (after increasing the library size) may have resulted in non-significant trends observed in SES. MNTD and $\beta$ NTI in ES chronosequence.

\section{Discussion}

To make ecological inferences using phylogenetic turnover requires a phylogenetic signal in the ecological niches of OTUs $[45,50]$. We detected significant phylogenetic signal across relatively short phylogenetic distances in all datasets. This suggests that more closely related bacterial taxa have more similar niche preferences related to soil $\mathrm{pH}$ [51]. Studying the phylogenetic turnover of closely related organisms can therefore be used to infer the underlying ecological processes in these successional soils [2]. These findings are in agreement with other studies, which found significant phylogenetic signal across relatively short phylogenetic distances across a broad range of ecosystems [2, $3,9,13]$.

Successional age was previously suggested as a primary factor governing bacterial community assembly along a glacial chronosequence [52]. It has been hypothesized that niches are spatially distributed over successional ages with more niches becoming available as succession proceeds, which may lead to a temporal transition from deterministic to stochastic assembly. On the other hand, it has also been hypothesized that niches fill through time, leading to a temporal transition from stochastic to deterministic assembly [13]. However, we found that successional age did not influence community assembly processes, which were 


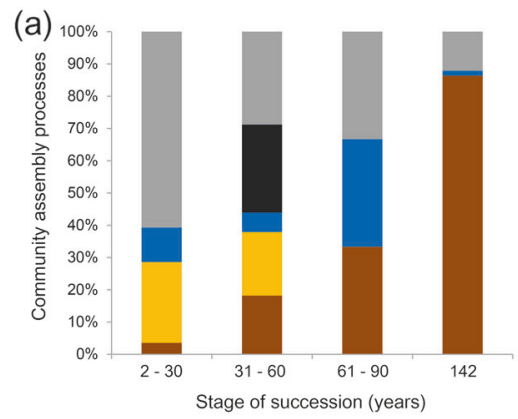

(b)
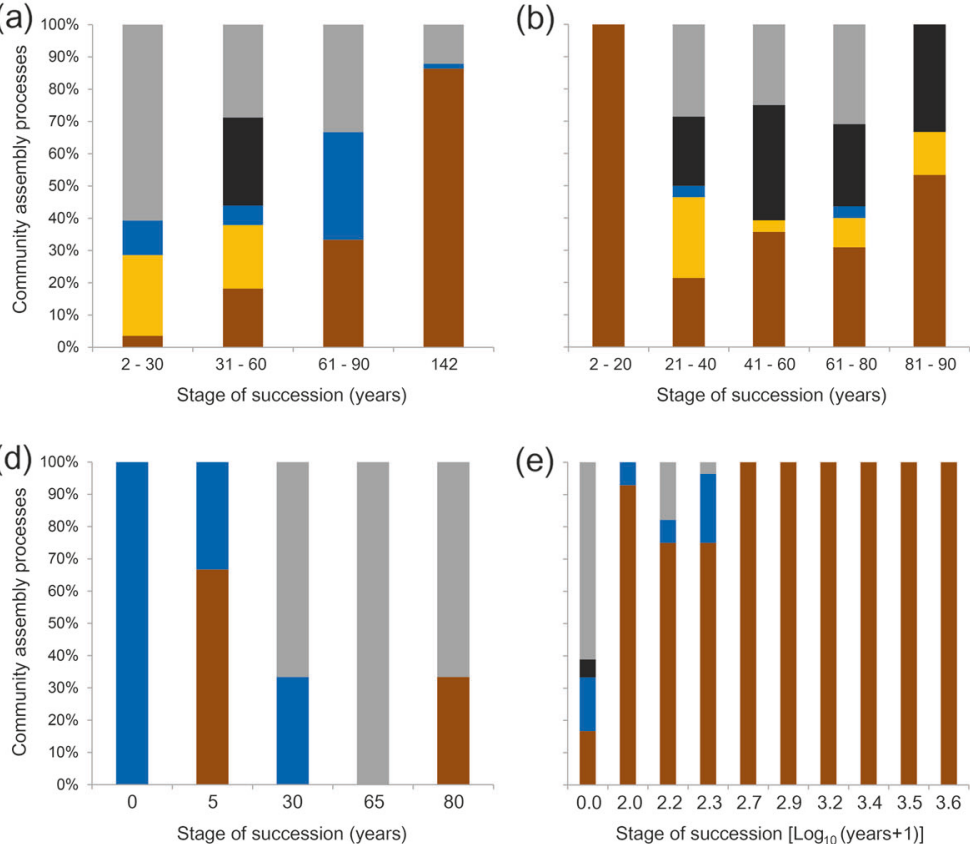

(e)

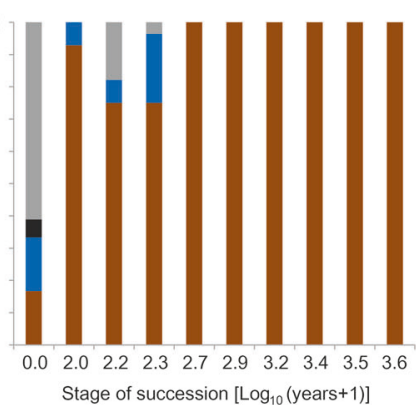

(c)

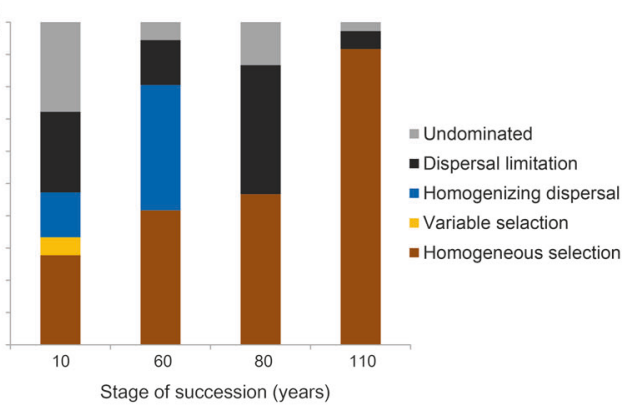

(f)

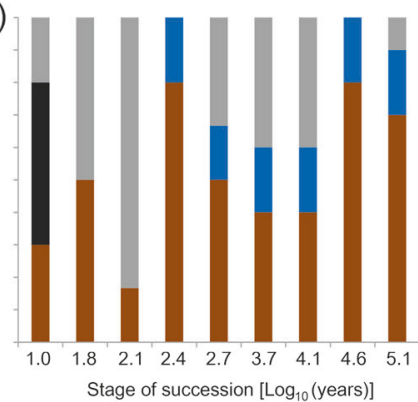

Fig. 6 The percent of turnover in bacterial community assembly governed primarily by various deterministic (homogeneous and variable selection) and stochastic processes (dispersal limitation and homogenizing dispersal), as well as the fraction that was not dominated by any single process, across successional ages in a AL, b ML, c DM, d ES, e FJ, and f SD chronosequences instead primarily associated with soil $\mathrm{pH}$. We found that extreme soil $\mathrm{pH}$ acts as a stringent environmental filter and leads to phylogenetic clustering, whereas the level of clustering is diminished under moderate $\mathrm{pH}$ regardless of the successional age. The relationship between soil $\mathrm{pH}$ and bacterial community assembly was also evident across a broad range of biomes in which successional age was not controlled (i.e., in the global dataset) [29, 31], which further supports our findings. Environmental filtering is a key determinant of community assembly [44], and this has been shown to result in phylogenetic clustering in bacterial communities [53]. These findings suggest that the relative influences of stochastic and deterministic community assembly processes can vary with successional age primarily because soil $\mathrm{pH}$ can vary with age.

Our results indicate that during succession the temporal trajectory of the stochastic-deterministic balance is not governed by time per se, but instead by the temporal trajectory of soil $\mathrm{pH}$. Examining the relative influence of stochastic processes (dispersal limitation, homogenizing dispersal, and the undominated fraction) in early successional soils that differed in $\mathrm{pH}$ provides further support to this inference. Specifically, $\mathrm{pH}$ was close to neutral in the early successional soils of AL, DM, SD, and FJ, and for these sites had relatively strong influences of stochastic assembly processes. The ML site provided the contrasting scenario in which $\mathrm{pH}$ was extreme in early successional soils, which was associated with a strong influence of deterministic assembly.
By complementing the within-community analysis (SES. MNTD) with between-community null model analysis using $\beta$ NTI we further showed that spatial processes (i.e., dispersal) were overwhelmed by deterministic selection imposed primarily by $\mathrm{pH}$. In support of this inference, we found $\beta$ NTI to be more strongly associated (partial Mantel coefficient) with soil $\mathrm{pH}$ than with spatial distance or other environmental distances in four sites (Tables S2 and S3). It is interesting to note that in AL and DM sites, spatial distance was significantly related to $\beta$ NTI after controlling for soil $\mathrm{pH}$ and other environmental differences. Although traditional analyses that link community dissimilarity metrics (e.g., Bray-Curtis) to spatial distances often infer influences of spatial processes, it has been argued conceptually and shown via simulation that spatial variation in $\beta$ NTI should not be driven by spatial processes [3, 9, 46]. Previous work linking $\beta$ NTI with spatial variables has therefore interpreted significant relationships between $\beta$ NTI and spatial distance as indicating that there are unmeasured, spatially autocorrelated environmental variables driving community composition through a selection-based mechanism, as opposed to dispersal [9]. This is because the variation in the magnitude of $\beta$ NTI is driven primarily by variation in deterministic processes, not by organismal dispersal [46, 47]. The reason is that the $\beta$ NTI null modeling approach is designed to detect differences in community composition that arise due to selection on organismal environmental optima, and is therefore not strongly influenced by dispersalbased community assembly. We therefore infer that 
ecological selection in the AL and DM sites is at least partially governed by environmental variables that were not measured, but that are spatially autocorrelated. It would be interesting in future efforts to attempt to identify these influential, yet unmeasured, variables.

Though soil $\mathrm{pH}$ is known to affect bacterial community composition and diversity at local [32, 54], regional [29, 30, 33], and global scales [55], it has not been clear how soil $\mathrm{pH}$ effects community assembly processes across these scales. Our results were consistent in both short-term and long-term successional datasets, and even in a heterogeneous global dataset in which successional age could not be controlled for [29, 31]. Our analyses spanned local to global scales and short- to long-term successional trajectories. The results showed consistent patterns across these spatial and temporal scales, indicating that soil $\mathrm{pH}$ mediates the relative influences of stochastic and deterministic processes across scales and across a broad range of ecosystems. Our results are conceptually consistent with the findings of Chase [56] on macroorganisms, which demonstrated that the presence of an extreme environmental filter such as drought results in strong deterministic selection. This finding has been further supported by other studies on macro-organisms [57, 58] and micro-organisms [59]. Combining those previous studies with the results observed here indicates that the same ecological principles govern community assembly processes across spatiotemporal scales, ecosystems, and taxonomic groups.

It is also interesting to consider whether there might be some link between the greater role of stochasticity in neutral $\mathrm{pH}$ soils and their greater diversity [29, 31, 33]. In an earlier study, Tripathi et al. [33] speculated the existence of a oneway evolutionary filter along $\mathrm{pH}$ gradients, whereby lineages of bacteria can easily branch from a more extreme $\mathrm{pH}$ environment to colonize - in evolutionary terms - a more neutral $\mathrm{pH}$ environment. This will tend to cause adapted lineages to accumulate in neutral $\mathrm{pH}$ environments, resulting in their greater diversity. The greater role of stochasticity, with weaker niche-based exclusion in a neutral $\mathrm{pH}$ environment, may assist the evolutionary arrival of lineages from more extreme $\mathrm{pH}$ environments.

\section{Conclusions and implications}

These results together with the previous conceptual model [13] lead us to propose a modified paradigm (Fig. 7), which describes how bacterial community assembly processes differ in relation to $\mathrm{pH}$, across successional soils. We hypothesize two possible scenarios for changes during succession. First, near-neutral pH in early successional soils will lead to more stochastic assembly and phylogenetically less clustered bacterial communities, and a shift in $\mathrm{pH}$ towards relatively extreme conditions in late successional
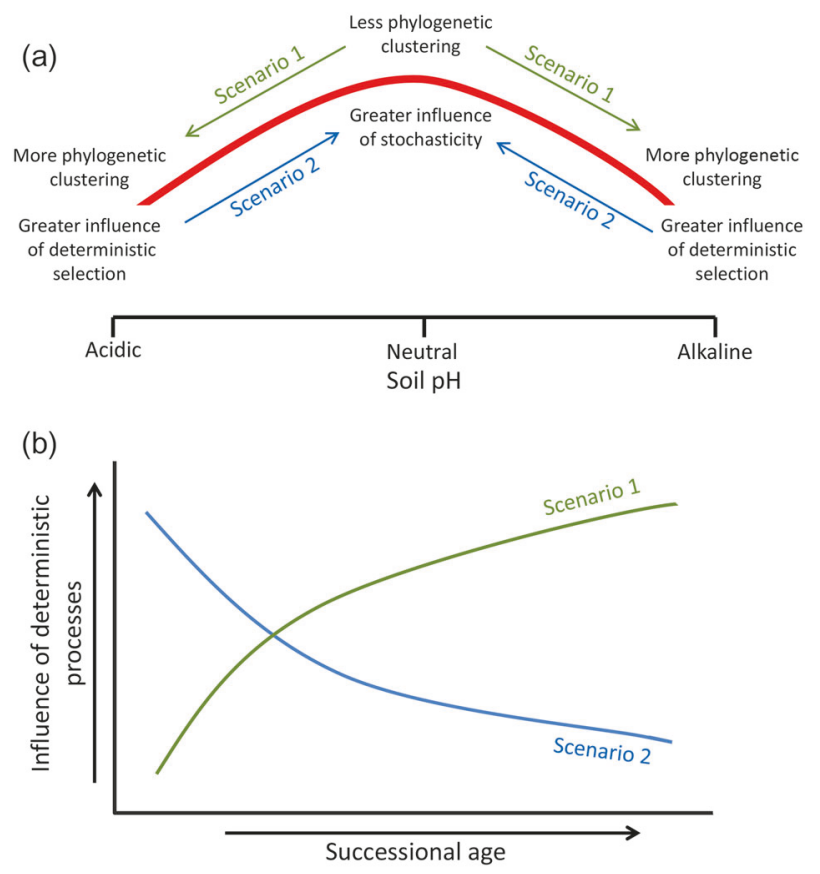

Fig. 7 Conceptual model showing two different possible scenarios in bacterial community assembly processes along successional soils with change in a soil $\mathrm{pH}$, and $\mathbf{b}$ temporal trajectory of the influence of deterministic processes for both scenarios

soils will lead to more deterministic assembly and phylogenetically more clustered bacterial communities. As an alternative, extreme $\mathrm{pH}$ in early successional soils will lead to deterministic assembly and phylogenetically more clustered communities, and progressive shifts in $\mathrm{pH}$ toward neutral conditions will lead to weaker selection, more stochasticity and phylogenetically less clustered bacterial communities. This conceptual model coupled with previous models provides a framework that could be experimentally tested in other successional environments.

Our broad examination of soils from around the world reinforces the view that the importance of deterministic vs. stochastic assembly of soil bacterial communities is significantly influenced by soil $\mathrm{pH}$. Thus, microbial communities in different types of soils are influenced by different, $\mathrm{pH}$-influenced balances between stochastic and deterministic community assembly processes. This variation in assembly processes may have implications for ecosystem function [60]. For example in neutral $\mathrm{pH}$ soils, biogeochemical function of the soil environment may be less predictable through time and perhaps more spatially variable due to the greater influence of stochasticity. In contrast, the biogeochemical function of soils with more extreme $\mathrm{pH}$ may be more consistent through time and more spatially homogeneous. Given the importance of soil processes for overall ecosystem function, this potential linkage betweencommunity assembly processes and soil processes should be evaluated both theoretically and experimentally across a 
broad range of systems. Nevertheless, although the relationship between $\mathrm{pH}$ and assembly processes is clear, it is important to bear in mind that $\mathrm{pH}$ accounts for a fairly small proportion $(17 \%)$ of total variation. Revealing additional factors that influence the balance between stochastic and deterministic assembly processes is an important topic for further investigations.

\section{Data accessibility}

The 16S rRNA gene sequence data of Midtre Lovénbreen Glacier chronosequence samples are deposited in the MGRAST server under project ID mgp21131 (http://meta genomics.anl.gov/linkin.cgi?project $=$ mgp21131). The $\mathrm{R}$ codes used for calculating SES.MNTD and $\beta$ NTI metrics are provided in the supplemental material.

Acknowledgements We thank Woosung Kim for help with the field work. This work was supported by the Korea Research Fellowship Program through the National Research Foundation of Korea funded by the Ministry of Science and ICT (NRF-2015H1D3A1066568), and partially supported by a National Research Foundation of Korea Grant from the Korean Government (MSIP) (NRF-2016M1A5A1901770). This research was also supported by Korea Polar Research Institute (PE16030 and PE17280). The research described in this paper was partially supported by the Microbiomes in Transition Initiative at Pacific Northwest National Laboratory. It was partially conducted under the Laboratory Directed Research and Development Program at PNNL, a multiprogram national laboratory operated by Battelle for the U.S. Department of Energy.

Author contributions BMT, JMA, and YKL conceived the study. DK performed field and laboratory work. BMT, JCS, and MK analyzed the sequence data. BMT, JCS, JMA, and YKL wrote the manuscript with feedback from all other authors. All authors read and approved the final manuscript.

\section{Compliance with ethical standards}

Conflict of interest The authors declare that they have no conflict of interest.

Open Access This article is licensed under a Creative Commons Attribution 4.0 International License, which permits use, sharing, adaptation, distribution and reproduction in any medium or format, as long as you give appropriate credit to the original author(s) and the source, provide a link to the Creative Commons license, and indicate if changes were made. The images or other third party material in this article are included in the article's Creative Commons license, unless indicated otherwise in a credit line to the material. If material is not included in the article's Creative Commons license and your intended use is not permitted by statutory regulation or exceeds the permitted use, you will need to obtain permission directly from the copyright holder. To view a copy of this license, visit http://creativecommons. org/licenses/by/4.0/.

\section{References}

1. Nemergut DR, Schmidt SK, Fukami T, O’Neill SP, Bilinski TM, Stanish LF, et al. Patterns and processes of microbial community assembly. Microbiol Mol Biol Rev. 2013;77:342-56.
2. Stegen JC, Lin X, Konopka AE, Fredrickson JK. Stochastic and deterministic assembly processes in subsurface microbial communities. ISME J. 2012;6:1653-64.

3. Wang J, Shen J, Wu Y, Tu C, Soininen J, Stegen JC, et al. Phylogenetic beta diversity in bacterial assemblages across ecosystems: deterministic versus stochastic processes. ISME J. 2013;7:1310-21.

4. Fargione J, Brown CS, Tilman D. Community assembly and invasion: an experimental test of neutral versus niche processes. Proc Natl Acad Sci USA. 2003;100:8916-20.

5. Chesson P. Mechanisms of maintenance of species diversity. Annu Rev Ecol Syst. 2000;31:343-66.

6. Hubbell SP. The unified neutral theory of biodiversity and biogeography. Princeton, NJ: Princeton University Press; 2001.

7. Chave J. Neutral theory and community ecology. Ecol Lett. 2004; 7:241-53.

8. Langenheder S, Székely AJ. Species sorting and neutral processes are both important during the initial assembly of bacterial communities. ISME J. 2011;5:1086-94.

9. Stegen JC, Lin X, Fredrickson JK, Chen X, Kennedy DW, Murray $\mathrm{CJ}$, et al. Quantifying community assembly processes and identifying features that impose them. ISME J. 2013;7:2069-79.

10. Zhou J, Deng Y, Zhang P, Xue K, Liang Y, Van Nostrand JD, et al. Stochasticity, succession, and environmental perturbations in a fluidic ecosystem. Proc Natl Acad Sci USA. 2014;111: E836-E845.

11. Chase JM, Myers JA. Disentangling the importance of ecological niches from stochastic processes across scales. Philos Trans R Soc Lond B Biol Sci. 2011;366:2351-63.

12. Chisholm RA, Pacala SW. Theory predicts a rapid transition from niche-structured to neutral biodiversity patterns across a speciation-rate gradient. Theor Ecol. 2011;4:195-200.

13. Dini-Andreote F, Stegen JC, van Elsas JD, Salles JF. Disentangling mechanisms that mediate the balance between stochastic and deterministic processes in microbial succession. Proc Natl Acad Sci USA. 2015;112:E1326-32.

14. Evans S, Martiny JB, Allison SD. Effects of dispersal and selection on stochastic assembly in microbial communities. ISME J. 2017;11:176-85.

15. Bardgett R. The biology of soil: a community and ecosystem approach. Oxford: Oxford University Press; 2005.

16. Guelland K, Hagedorn F, Smittenberg R, Göransson H, Bernasconi S, Hajdas I, et al. Evolution of carbon fluxes during initial soil formation along the forefield of Damma glacier, Switzerland. Biogeochemistry. 2013;113:545-61.

17. Matthews JA. The ecology of recently deglaciated terrain: a geoecological approach to glacier forelands. Cambridge: Cambridge Univ. Press; 1992.

18. Menge DN, Hedin LO. Nitrogen fixation in different biogeochemical niches along a 120 000-year chronosequence in New Zealand. Ecology. 2009;90:2190-201.

19. Turner BL, Condron LM, Richardson SJ, Peltzer DA, Allison VJ. Soil organic phosphorus transformations during pedogenesis. Ecosystems. 2007;10:1166-81.

20. Dini-Andreote F, de Cássia Pereira e Silva M, Triado-Margarit X, Casamayor EO, van Elsas JD, Salles JF. Dynamics of bacterial community succession in a salt marsh chronosequence: evidences for temporal niche partitioning. ISME J. 2014;8:1989-2001.

21. Brown SP, Jumpponen A. Contrasting primary successional trajectories of fungi and bacteria in retreating glacier soils. Mol Ecol. 2014;23:481-97.

22. Kim M, Jung JY, Laffly D, Kwon HY, Lee YK. Shifts in bacterial community structure during succession in a glacier foreland of the high Arctic. FEMS Microbiol Ecol. 2017;93:fiw213.

23. Castle SC, Nemergut DR, Grandy AS, Leff JW, Graham EB, Hood E, et al. Biogeochemical drivers of microbial community 
convergence across actively retreating glaciers. Soil Biol Biochem. 2016;101:74-84.

24. Rime T, Hartmann M, Brunner I, Widmer F, Zeyer J, Frey B. Vertical distribution of the soil microbiota along a successional gradient in a glacier forefield. Mol Ecol. 2015;24:1091-108.

25. Schüette UM, Abdo Z, Foster J, Ravel J, Bunge J, Solheim B, et al. Bacterial diversity in a glacier foreland of the high Arctic. Mol Ecol. 2010;19:54-66.

26. Jangid K, Whitman WB, Condron LM, Turner BL, Williams MA. Soil bacterial community succession during long-term ecosystem development. Mol Ecol. 2013;22:3415-24.

27. Williams MA, Jangid K, Shanmugam SG, Whitman WB. Bacterial communities in soil mimic patterns of vegetative succession and ecosystem climax but are resilient to change between seasons. Soil Biol Biochem. 2013;57:749-57.

28. Ferrenberg S, O’Neill SP, Knelman JE, Todd B, Duggan S, Bradley $\mathrm{D}$, et al. Changes in assembly processes in soil bacterial communities following a wildfire disturbance. ISME J. 2013;7:1102-11.

29. Chu H, Fierer N, Lauber CL, Caporaso J, Knight R, Grogan P. Soil bacterial diversity in the Arctic is not fundamentally different from that found in other biomes. Environ Microbiol. 2010;12:2998-3006.

30. Griffiths RI, Thomson BC, James P, Bell T, Bailey M, Whiteley AS. The bacterial biogeography of British soils. Environ Microbiol. 2011;13:1642-54.

31. Lauber CL, Hamady M, Knight R, Fierer N. Pyrosequencingbased assessment of soil $\mathrm{pH}$ as a predictor of soil bacterial community structure at the continental scale. Appl Environ Microbiol. 2009;75:5111-20.

32. Rousk J, Bååth $\mathrm{E}$, Brookes $\mathrm{PC}$, Lauber CL, Lozupone $\mathrm{C}$, Caporaso JG, et al. Soil bacterial and fungal communities across a pH gradient in an arable soil. ISME J. 2010;4:1340-51.

33. Tripathi BM, Kim M, Singh D, Lee-Cruz L, Lai-Hoe A, Ainuddin A, et al. Tropical soil bacterial communities in Malaysia: $\mathrm{pH}$ dominates in the equatorial tropics too. Microb Ecol. 2012;64:474-84.

34. Dong K, Tripathi B, Moroenyane I, Kim W, Li N, Chu H, et al. Soil fungal community development in a high Arctic glacier foreland follows a directional replacement model, with a midsuccessional diversity maximum. Sci Rep. 2016;6:26360.

35. Herlemann DP, Labrenz M, Jürgens K, Bertilsson S, Waniek JJ, Andersson AF. Transitions in bacterial communities along the $2000 \mathrm{~km}$ salinity gradient of the Baltic Sea. ISME J. 2011;5:1571-9.

36. Schloss PD, Westcott SL, Ryabin T, Hall JR, Hartmann M, Hollister EB, et al. Introducing mothur: open-source, platformindependent, community-supported software for describing and comparing microbial communities. Appl Environ Microbiol. 2009;75:7537-41.

37. Schirmer M, Ijaz UZ, D’Amore R, Hall N, Sloan WT, Quince C. Insight into biases and sequencing errors for amplicon sequencing with the Illumina MiSeq platform. Nucleic Acids Res. 2015;43: e37.

38. Masella AP, Bartram AK, Truszkowski JM, Brown DG, Neufeld JD. PANDAseq: paired-end assembler for Illumina sequences. BMC Bioinformatics. 2012;13:31.

39. Edgar RC, Haas BJ, Clemente JC, Quince C, Knight R. UCHIME improves sensitivity and speed of chimera detection. Bioinformatics. 2011;27:2194-2200.
40. Price MN, Dehal PS, Arkin AP. FastTree 2-approximately maximum-likelihood trees for large alignments. PLoS ONE. 2010;5: e9490.

41. Huisman J, Olff H, Fresco LFM. A hierarchical set of models for species response analysis. J Veg Sci. 1993;4:37-46.

42. Jansen F, Oksanen J. How to model species responses along ecological gradients-Huisman-Olff-Fresco models revisited. J Veg Sci. 2013;24:1108-17.

43. Kembel SW, Cowan PD, Helmus MR, Cornwell WK, Morlon H, Ackerly DD, et al. Picante: R tools for integrating phylogenies and ecology. Bioinformatics. 2010;26:1463-4.

44. Webb CO, Ackerly DD, McPeek MA, Donoghue MJ. Phylogenies and community ecology. Annu Rev Ecol Syst. 2002;33:475-505.

45. Fine PV, Kembel SW. Phylogenetic community structure and phylogenetic turnover across space and edaphic gradients in western Amazonian tree communities. Ecography. 2011;34:552-65.

46. Stegen JC, Lin X, Fredrickson JK, Konopka AE. Estimating and mapping ecological processes influencing microbial community assembly. Front Microbiol. 2015;6:370.

47. Hardy OJ. Testing the spatial phylogenetic structure of local communities: statistical performances of different null models and test statistics on a locally neutral community. J Ecol. 2008;96:914-26.

48. Wood S. mgcv: Mixed GAM Computation Vehicle with GCV/ AIC/REML smoothness estimation. R package v. 1.8-17. 2012. https://CRAN.R-project.org/package $=$ mgcv.

49. Goslee SC, Urban DL. The ecodist package for dissimilaritybased analysis of ecological data. J Stat Softw. 2007;22:1-19.

50. Cavender-Bares J, Kozak KH, Fine PV, Kembel SW. The merging of community ecology and phylogenetic biology. Ecol Lett. 2009;12:693-715

51. Losos JB. Phylogenetic niche conservatism, phylogenetic signal and the relationship between phylogenetic relatedness and ecological similarity among species. Ecol Lett. 2008;11:995-1003.

52. Brown SP, Jumpponen A. Phylogenetic diversity analyses reveal disparity between fungal and bacterial communities during microbial primary succession. Soil Biol Biochem. 2015;89:52-60.

53. Horner-Devine MC, Bohannan BJ. Phylogenetic clustering and overdispersion in bacterial communities. Ecology. 2006;87: S100-8.

54. Tripathi BM, Lee-Cruz L, Kim M, Singh D, Go R, Shukor NA, et al. Spatial scaling effects on soil bacterial communities in Malaysian tropical forests. Microb Ecol. 2014;68:247-58.

55. Fierer N, Jackson RB. The diversity and biogeography of soil bacterial communities. Proc Natl Acad Sci USA. 2006;103:626-31.

56. Chase JM. Drought mediates the importance of stochastic community assembly. Proc Natl Acad Sci USA. 2007;104:17430-4.

57. Helmus MR, Keller WB, Paterson MJ, Yan ND, Cannon CH, Rusak JA. Communities contain closely related species during ecosystem disturbance. Ecol Lett. 2010;13:162-74.

58. Myers JA, Harms KE. Seed arrival and ecological filters interact to assemble high-diversity plant communities. Ecology. 2011;92:676-86.

59. Valverde A, Makhalanyane TP, Cowan DA. Contrasting assembly processes in a bacterial metacommunity along a desiccation gradient. Front Microbiol. 2014;5:668.

60. Graham EB, Stegen JC. Dispersal-based microbial community assembly decreases biogeochemical function. Processes. 2017;5:65. 